\title{
Radiation damage in tungsten from cascade overlap with voids and vacancy clusters
}

\section{Fellman, A.}

2019-10-09

Fellman , A , Sand , A E , Byggmästar , J \& Nordlund , K 2019 , ' Radiation damage in tungsten from cascade overlap with voids and vacancy clusters ', Journal of Physics.

Condensed Matter , vol. 31 , no. 40 , 405402 . https://doi.org/10.1088/1361-648X/ab2ea4

http://hdl.handle.net/10138/304669

https://doi.org/10.1088/1361-648X/ab2ea4

cc_by_nc_sa

Downloaded from Helda, University of Helsinki institutional repository.

This is an electronic reprint of the original article.

This reprint may differ from the original in pagination and typographic detail.

Please cite the original version. 


\title{
Radiation damage in tungsten from cascade overlap with voids and vacancy clusters
}

\author{
A. Fellman ${ }^{\mathrm{a}}$, A. E. Sand ${ }^{\mathrm{a}, *}$, J. Byggmästar ${ }^{\mathrm{a}}$, K. Nordlund ${ }^{\mathrm{a}}$ \\ ${ }^{a}$ Department of Physics, P.O. Box 43, FI-00014 University of Helsinki, Finland
}

\begin{abstract}
We have performed a systematic molecular dynamics investigation of the effects of overlap of collision cascades in tungsten with pre-existing vacancy-type defects. In particular, we focus on the implications for fusion neutron irradiated tungsten in relation to comparisons with damage production under ion irradiation conditions. We find that overlap of a cascade with a vacancy-type defect decreases the number of new defects with roughly the same functional dependence as previously shown for interstitial clusters. We further find that different mechanisms govern the formation of dislocation loops, resulting in different Burgers vectors, depending on the degree of overlap between the cascade and the defect. Furthermore, we show that overlapping cascades consistently decrease the size of the pre-existing defect. We also observe void-induced cascade splitting at energies far below the subcascade splitting threshold in tungsten. The impact of these mechanisms on radiation damage accumulation and dose rate effects are discussed.
\end{abstract}

Keywords:

tungsten, radiation damage, molecular dynamics, voids, cascade overlap

\section{Introduction}

Tungsten (W) is currently considered to be a top candidate as a structural and plasma-facing material for fusion reactors [1]. Tungsten has many favourable properties, making it well suited for the extreme conditions in a fusion reactor, including high melting point, good thermal conductivity, and ability to resist erosion $[1,2,3]$. In fusion reactors, the materials are exposed to both high temperatures and significant neutron radiation doses, which over time cause the degradation of the materials' mechanical and physical properties. To determine the feasibility of using $\mathrm{W}$ in future fusion reactors, the effects of radiation damage are of great interest.

\footnotetext{
* Corresponding author

Email address: andrea.sand@helsinki.fi (A. E. Sand)
} 
When a neutron collides elastically with an atom in a material, it causes the atom to recoil. This primary knock-on atom (PKA) may then in turn collide with neighbouring atoms, causing them to subsequently collide with their neighbours [4]. This process is called a collision cascade. Primary radiation damage resulting from collision cascades has been studied quite extensively with molecular dynamics (MD) methods, and the process of formation of the primary damage from individual cascades is fairly well understood [5, 4]. However, when a material is subjected to continued irradiation, eventually a collision cascade will overlap with a defect already present in the material before the cascade. The effects of overlap between cascades and pre-existing defects have been studied much less than individual cascades in pristine materials. Although there have been some studies of cascades interacting with pre-existing damage $[6,7,8,9]$ and multiple overlapping cascades $[10,11,12]$, the possible number of overlap types is so large, that the understanding is yet far from comprehensive..

It has been observed experimentally that defects in iron appeared only after a threshold dose of irradiation was met [13]. Later, it was also demonstrated that the amount of visible defects increased non-linearly with increased radiation dose [14]. These findings imply that the effects of cascade overlap have an impact on the observed defects. Early MD simulations, on the other hand, have shown that the amount of point defects produced decreases, if the collision cascade overlaps with pre-existing defects [15]. Most of the current literature on the effects of overlap in simulations focuses on iron, while effects in tungsten have been studied considerably less.

Recently, we have investigated the effects of overlap both in $\alpha$-Fe and $\mathrm{W}$, using MD simulations $[16,17]$. We showed that overlapping cascades have a significant impact on the morphology of the final defects, in addition to the effects on defect formation efficiency. In a systematic study of cascade overlap on interstitial defects, we found that the number of new Frenkel pairs produced by a cascade is significantly reduced as a result of the cascade overlap in both iron and tungsten [18]. In addition, we also proposed an analytical model for predicting the number of Frenkel pairs produced as a function of the degree of overlap between the cascade and the pre-existing defect.

In this work, we study the effects of cascades overlapping with vacancy-type defects in tungsten using molecular dynamics. We restrict this study to defects that have radii smaller than the cascade radius. We consider pre-existing voids and vacancy-type dislocation loops with different Burgers vectors, while also comparing the predictions of different interatomic potentials. Unlike the simulations performed in [17], we have not used the end product of a previous cascade as the initial damage. Instead, we have manually created well defined vacancy-type defects; this allows a systematic study of how the size and morphology of the pre-existing defect affects the damage produced by cascade overlap. These pre-existing defects represent low energy defect configurations, that are expected to be present in, for example, nuclear reactors at operating temperatures. They are also the defects that standard object kinetic Monte Carlo (OKMC) models of long term radiation effects describe [19, 20, 21], so that this systematic MD study provides direct input for multi-scale modelling 
of radiation damage accumulation.

\section{Methods and analysis}

\subsection{Simulation methods}

We introduced defects in cubic simulation cells of bcc tungsten by removing atoms in the center of the cell to create vacancies. Voids were created in a lattice with the major crystallographic directions aligned with the cell sides, by removing atoms that were within a given radius. Dislocation loops were created by first removing atoms in a circular plane corresponding to a Burgers vector of $\langle 100\rangle$ or $1 / 2\langle 111\rangle$, after which the region was compressed to form a dislocation loop. The lattice in these simulation cells was oriented so that the Burgers vector in each case was parallel to one side of the cell. The cells were then relaxed at $300 \mathrm{~K}$. During the relaxation the temperature was controlled using a Berendsen thermostat [22], and a barostat set to $0 \mathrm{kBar}$. In Table 1, we list the different defects types and sizes that were used in this work, in terms of the numbers of vacancies in each cluster, and the corresponding cluster radius.

\begin{tabular}{lcc}
\hline \hline Defect type & $N_{\text {vac }}$ & $r_{\mathrm{d}}(\AA)$ \\
\hline Void & $(59,259,893,2085)$ & $(6,10,15,20)$ \\
$\langle 100\rangle$ & $(101,197)$ & $(13,18)$ \\
$1 / 2\langle 111\rangle$ & $(109,199)$ & $(14,19)$ \\
\hline \hline
\end{tabular}

Table 1: The pre-existing vacancy-type defects used in this study, their sizes in terms of numbers of vacancies $N_{\mathrm{Vac}}$, and the corresponding defect radius $r_{d}$.

Cascade simulations were then performed on the relaxed systems. The primary knock-on atom (PKA) was chosen by first generating a random angle, with angles uniformly distributed over the sphere, and then selecting an atom in that direction, at a given distance from the center of the cell.

The PKA was then given a velocity corresponding to the desired kinetic energy, directed towards the center of the cell. For $10 \mathrm{keV}$ PKAs, the simulation cells contained approximately 300000 atoms. In $30 \mathrm{keV}$ simulations the system size was approximately 1000000 atoms. The simulation cell was given periodic boundaries, and if the kinetic energy of an atom at the border exceeded a $10 \mathrm{eV}$ threshold, the cascade was considered to have extended over the borders of the simulation cell, in which case the simulation was aborted, to avoid selfinteraction of the cascade. For each defect type and size, a total of at least 800 individual cascade simulations were run, with the PKAs initiated from between $8 \AA$ to $80 \AA$ from the center of the defect.

The simulations were performed with the MD code PARCAS [23]. We used three different embedded-atom method (EAM) potentials [24], namely the potential by Derlet et al.[25] and modified by Björkas et al.[26](hereafter referred to as DND-BN), the Finnis-Sinclair potential by Ackland et al.[27], modified 
by Zhong et al.[28](hereafter referred to as AT-ZN), and finally the more recent potential by Marinica et al.[29] and modified by Sand et al.[30] (hereafter referred to as M4-S). The AT-ZN potential has been widely used for cascade simulations, while the DND-BN potential has been shown to give cascade damage results in good agreement with experiment [31,32]. The M4-S potential, on the other hand, has been extensively fitted to liquid configurations, and gives a good representation of line defects.

\subsection{Analysis}

The results of the collision cascade simulations were analysed using the tools provided with OVITO [33]. OVITO's Wigner-Seitz analysis was used to calculate the number and locations of interstitials and vacancies in the system. The dislocation analysis algorithm DXA [34] was used to determine whether the preexisting dislocation loops survived the cascade, whether they had been altered, or whether new dislocations had been formed. We also performed a cluster analysis to determine the numbers of interstitial and vacancy clusters in the systems, and their sizes. The cut-off distance for the vacancy cluster analysis was chosen to be halfway between the second nearest neighbour and third nearest neighbours, $\left(r_{2 N N}+r_{3 N N}\right) / 2$. For interstitials, we used the midway-point between the third nearest and fourth nearest neighbours, $\left(r_{3 N N}+r_{4 N N}\right) / 2$.

To determine the collision cascade center, we calculated the center of mass position of the liquid atoms existing during the period of maximal extent of the hot cascade core, with each liquid atom weighted according to its kinetic energy. We defined an atom as liquid when the average kinetic energy of the atom and its neighbors exceeds the melting point of the potential [35].

The data are divided into bins with respect to the distance between the cascade center and defect center. A statistical mean is calculated for the given bin, and the mean value and the bin's mean position is then plotted. The error is calculated by the standard error of the values in the bin. Since the individual cascades are independent of one another, the final defect count follows a Poisson distribution. As a result, especially for the low defect count in the case of full overlap by the second cascade, the mean and median values differ slightly. This is illustrated in Figure 1, which also shows the peak of the Poisson distribution fitted to the data. We expect that most applications of these data as input to larger scale simulations will use only a single value, and disregard fluctuations. Hence, in what follows, we report only the mean values. We reject the last bin, included for completeness in Figure 1, since it generally contains too few data points. 


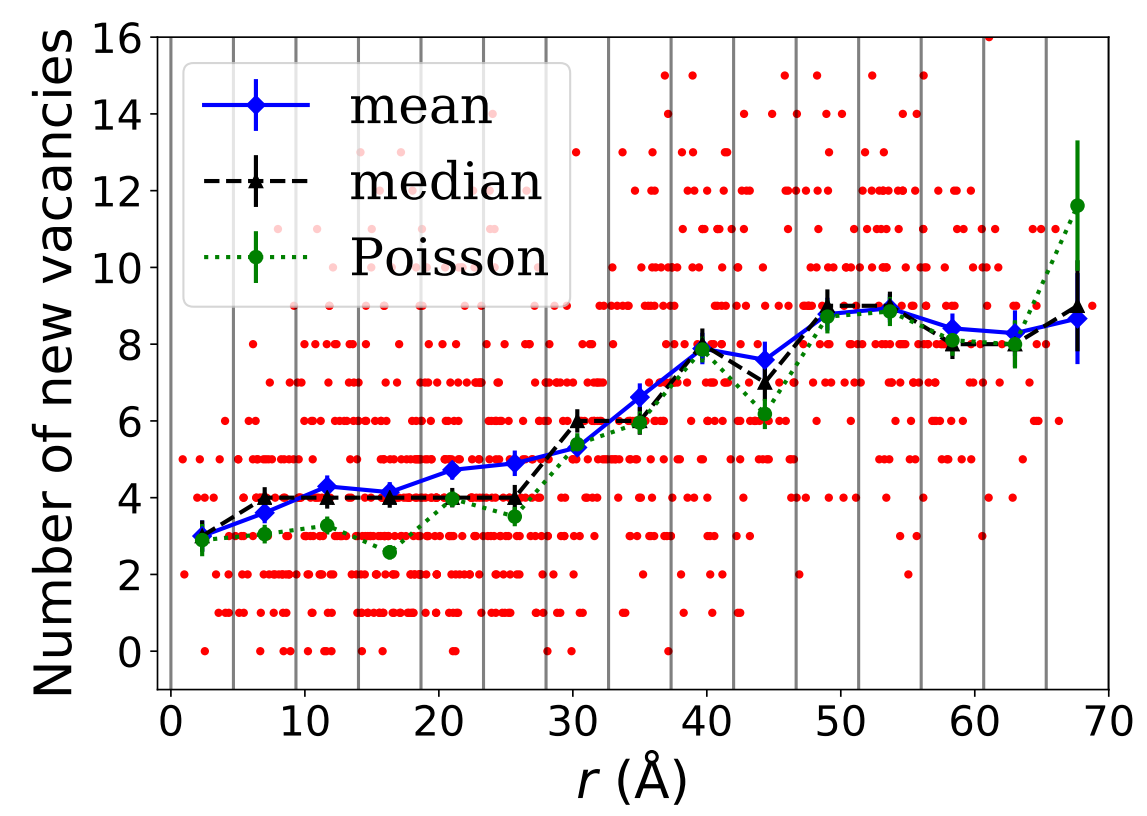

Figure 1: Binning and statistical representation of an example system (pre-existing $\langle 100\rangle$ loop, $N_{\text {Vac }}=101$ ), showing the mean and median values of the raw data, as well as the peak value of a fitted Poisson distribution for the data in each bin.

\section{Results}

\subsection{Defect numbers at full overlap}

We find that the production of new defects decreases when the cascade develops in a region with a pre-existing vacancy-type defect. This observation agrees with previous results showing a decrease in the number of defects produced in pre-damaged material $[15,18]$. In Figure 2, we plot the number of new defects resulting from cascades overlapping with voids of increasing size. In these simulations, the PKA was initiated $15 \AA$ from the void center, to achieve maximal overlap. The number of new vacancies decreased with increasing size of the pre-existing vacancy cluster.

The number of new vacancies $N_{\text {def }}$, as a function of void size, is well represented by

$$
N_{\text {def }}=N_{0}-a \ln N_{\text {vac }},
$$

where $N_{0}$ is the (potential dependent) average number of defects formed from an isolated cascade in the perfect crystal, and $N_{\text {vac }}$ is the number of vacancies in the pre-existing void. For this study, we determined $N_{0}$ from 200 cascade simulations with each potential, initiated in a perfect lattice at $300 \mathrm{~K}$. We find that $a=0.6$ yields a good fit to the data for void overlap with all potentials, 


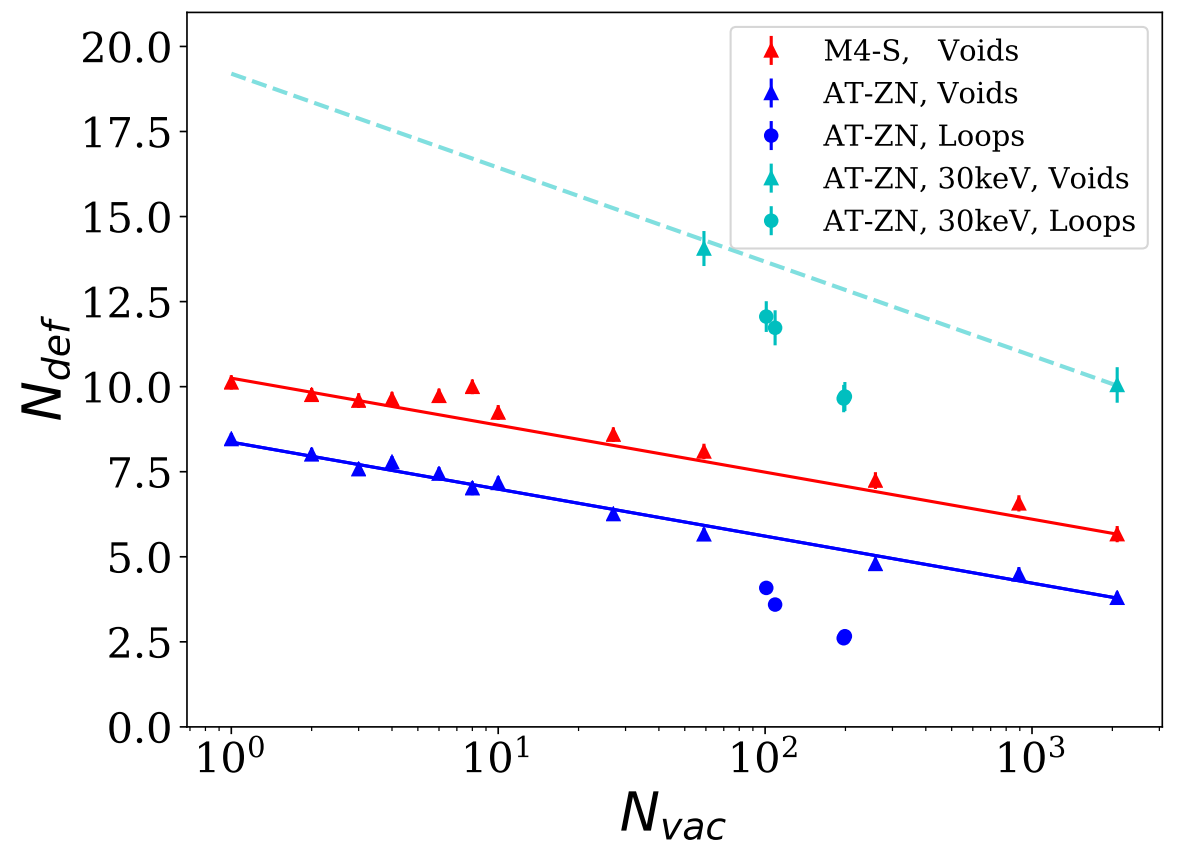

Figure 2: Numbers of new Frenkel pairs produced as a function of the size of the pre-existing $\operatorname{defect}\left(N_{\mathrm{Vac}}\right)$, using the AT-ZN potential. Each data point represents a statistical mean from 200 (100) simulations for $10 \mathrm{keV}(30 \mathrm{keV})$ PKAs, with the PKA initiated $15 \AA$ from the defect center.

despite the fact that the M4-S potential consistently predicts a slightly higher number of defects, compared to the other potentials. However, direct cascade overlap with pre-existing dislocation loops yields fewer new defects than overlap with voids. A full dependence of this value on loop size cannot be determined, since small vacancy-type dislocation loops are not stable at $300 \mathrm{~K}$, but rather open up to form so called "open vacancy loops" [36], which are not expected to occur naturally, due to preference for the void structure. On the other hand, $10 \mathrm{keV}$ cascades are barely large enough to envelop the larger, stable loops considered here, and a further increase in size would involve defects larger than the extent of the cascade.

We further note that this function also depends on cascade energy. A full investigation of the PKA energy dependence will be the focus of future work. Here, we note that the limited data we have for $30 \mathrm{keV}$ PKAs suggests that equation (1) may be valid in general for void overlap, with dislocation loops causing a stronger decrease in the number of new defects than voids, for a given PKA energy. The origin of this difference between loops and voids is considered in the following section. 


\subsection{Damage as a function of defect - cascade separation}

In the case of full overlap between the cascade and a dislocation loop, i.e. where the sum of the defect radius and the defect-cascade separation is less than the cascade radius, the number of new vacancies that are produced is at a minimum. When the cascade develops further away from the defect center, and the degree of overlap decreases, the number of new vacancies increases, up to the point where the pre-existing defect is fully outside of the cascade radius, and the number of new vacancies converges to the value for cascades in pure crystalline $\mathrm{W}$ without defects. Figure 3 shows the effect of the degree of cascade overlap on the production of new defects. The different potentials agree qualitatively, but differ in the overall numbers of new vacancies. For the larger loops, partial overlap occurs out to a larger separation distance, hence the region over which the number of new defects is reduced is correspondingly larger.

In figures 3-4, we have plotted a slightly modified version of the function introduced in Ref. [18]:

$$
N= \begin{cases}N_{\mathrm{def}} & , r<r_{\mathrm{d}} \\ N_{0}-\left(N_{0}-N_{\mathrm{def}}\right) \exp \left[-\frac{\left(r-r_{\mathrm{d}}\right)^{2}}{r_{\mathrm{c}}^{2}}\right] & , r \geq r_{\mathrm{d}},\end{cases}
$$

where $r_{\mathrm{d}}$ is the radius of the pre-existing defect (see table 1 ), and $r_{\mathrm{c}}$ is the cascade radius. We define $N_{\text {def }}$ for $10 \mathrm{keV}$ cascades using Equation (1) (see Figure 2 for relation to the data). Tables 1 and 2 list the values used here for the parameters in Eq. (2). We find that the function, developed for interstitial defect overlap, also fits the data for overlap with vacancy-type dislocation loops quite well. The main discrepancy is seen in the region of partial overlap, where the model slightly overestimates defect numbers.

\begin{tabular}{lccc}
\hline \hline Potential & $E_{\mathrm{PKA}}$ & $N_{0}$ & $r_{\mathrm{c}}[18]$ \\
\hline AT-ZN & $10 \mathrm{keV}$ & $8.37 \pm 0.1884$ & 19.2 \\
DND-BN & $10 \mathrm{keV}$ & $8.92 \pm 0.2012$ & 18.8 \\
M4-S & $10 \mathrm{keV}$ & $10.25 \pm 0.212$ & 17.7 \\
\hline \hline
\end{tabular}

Table 2: Values for the different potentials of number of defects from cascades in perfect crystal $N_{0}$, and the cascade radius $r_{\mathrm{c}}$ (taken from Ref. [18]) used in Equation (2) for $10 \mathrm{keV}$ cascades, plotted in Figures 3-4. All values pertain to cascades simulated at $300 \mathrm{~K}$.

The behaviour of cascades overlapping with voids differs slightly from that of overlap with vacancy-type loops, except for the very smallest void size. When the center of the cascade is close to the center of a void of significant size, the number of new vacancies is not at a minimum (see Figure 4). For increased separation between cascade and void center, the number of new defects decreases, until at larger separation the value again increases, towards that of isolated cascades in pure crystal. We note that this effect is not captured by Equation (2), and in particular that $N_{0}$ is determined here by the full overlap behavior, and hence Equation (2) clearly overestimates the defect numbers in the region 


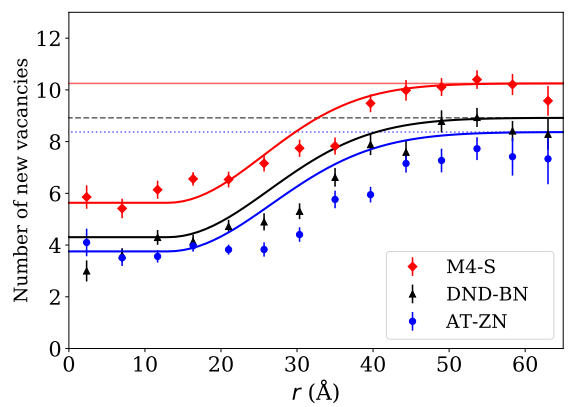

(a) $\langle 100\rangle, N_{\text {vac }}=101$

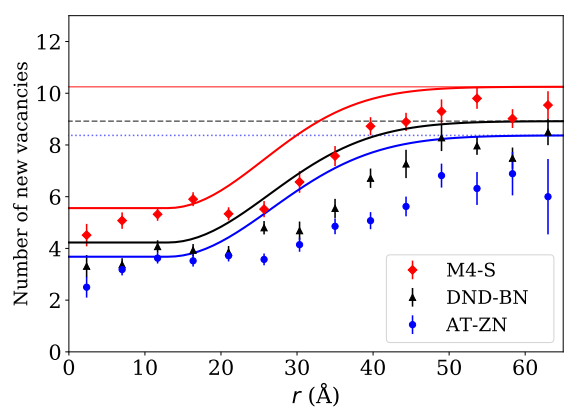

(c) $1 / 2\langle 111\rangle, N_{\text {vac }}=109$

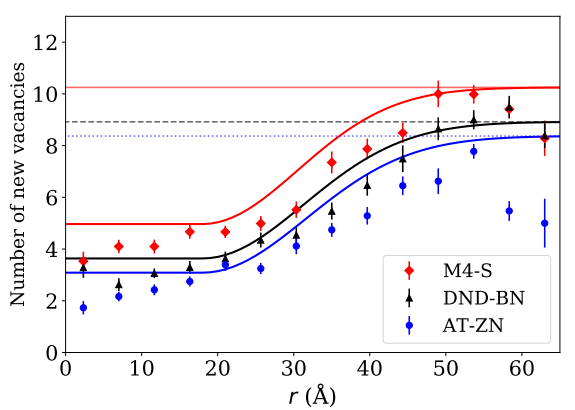

(b) $\langle 100\rangle, N_{\text {vac }}=197$

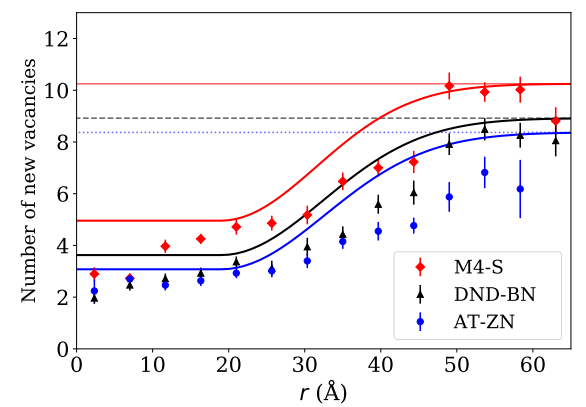

(d) $1 / 2\langle 111\rangle, N_{\text {vac }}=199$

Figure 3: Numbers of new defects from cascades overlapping with dislocation loops of different types (Burgers vectors $\langle 100\rangle$ and $1 / 2\langle 111\rangle$ ) and sizes $\left(N_{\mathrm{vac}}\right)$, plotted as a function of the distance $r$ between defect center and cascade center. All cascades had $10 \mathrm{keV}$ PKA-energy, and were simulated at $300 \mathrm{~K}$. 
of partial overlap of voids with the current choice of parameters. However, the prediction is nevertheless useful for providing a first approximation to the damage rates in pre-damaged material.

Closer inspection of the cascade region in cases where the cascade was centered on the void showed that often the presence of the void had caused the cascade to split, and develop in fully separate regions around the void. This void-induced cascade splitting is illustrated in Figure 5(a). The two parts of the illustrated cascade are largely unaffected by the void (in turn having little effect on the void itself). Due to the possibility, but not necessity, of the cascade splitting, the numbers of new defects at full overlap show strong variation, and the uncertainty in the mean value is large. When the cascade only partially overlaps with the void, as in Figure 5(b), the decrease in the number of new defects is the strongest, and simultaneously the spread in the data is smaller. As with dislocation loops, when the cascade is far enough from the defect, it has no influence on the defect (Figure 5(c)), and the defect, in turn, does not affect the numbers of new vacancies produced by the cascade.

\subsection{Effect of PKA energy}

We have investigated the effect of PKA energy using the AT-ZN potential, for a selection of the pre-existing defects. Increasing the PKA energy to $30 \mathrm{keV}$ increases the cascade radius, which in turn means that partial overlap with the defect occurs for a larger separation distance between cascade center and defect center, and hence the region where defect numbers are reduced is larger. A more energetic cascade also increases the number of defects produced. Figure 6 shows the number of new vacancies formed in $30 \mathrm{keV}$ cascades overlapping with different vacancy-type defects at different separation distances. For the largest defect sizes, we did not extend the overlap distance of $30 \mathrm{keV}$ cascades to the fully non-overlapping situation, since this would have required an excessively large simulation box, considering the large number of individual simulations needed for adequate statistics. It is, however, clear that the number of new defects will converge to the value in a perfect crystal at larger separation distance, as for the $10 \mathrm{keV}$ cascades. Overall, the behaviour for $10 \mathrm{keV}$ and $30 \mathrm{keV}$ cascades is qualitatively similar.

\subsection{Survival of the pre-existing defect}

We investigate the effect of cascade overlap on void and loop growth by analyzing the sizes of the resulting vacancy clusters in relation to the pre-existing voids and loops. The final clusters are products of both the effect of the formation of new defects, as well as the tendency of the cascade to either split or cluster the final vacancy population. Overall, we find that the vacancy clusters decrease in size, despite the formation of additional vacancies as a result of the cascade. This is in clear contrast to the observations of interstitial defects, where a slight increase in defect size was observed when cascades partially overlap with interstitial loops [18].

The decrease in cluster sizes for loops and voids are shown in Figures $7 \&$ 8 , respectively, both in terms of absolute vacancy numbers, and as a fractional 


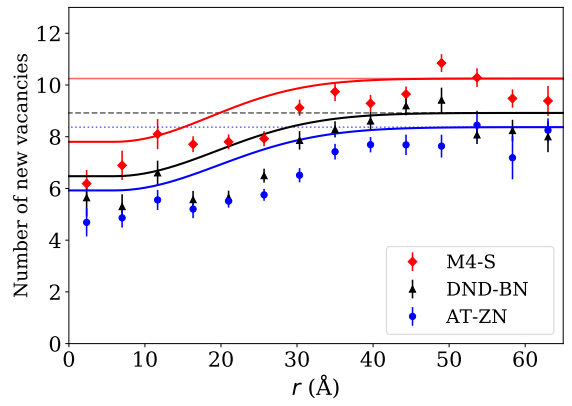

(a) void, $N_{\text {vac }}=59$

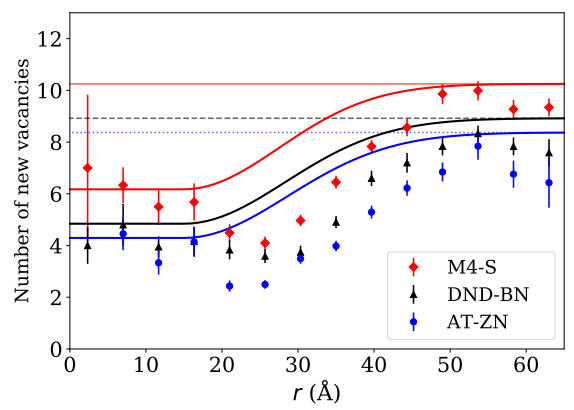

(c) void, $N_{\text {vac }}=893$

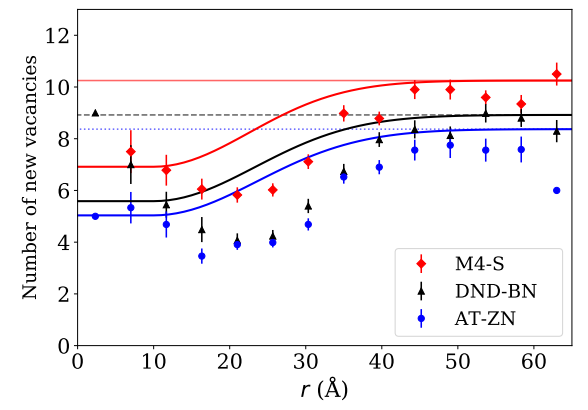

(b) void, $N_{\text {vac }}=259$

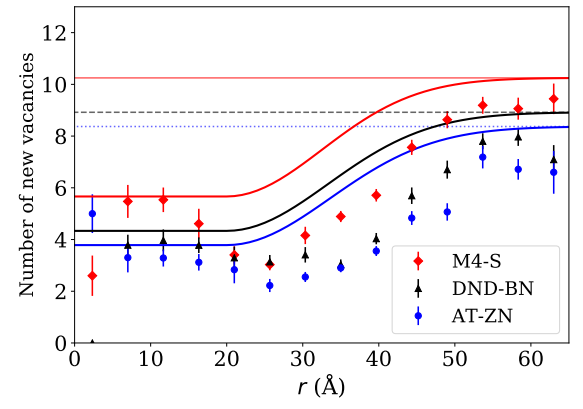

(d) void, $N_{\text {vac }}=2085$

Figure 4: Number of new defects created in $10 \mathrm{keV}$ cascade simulations on pre-existing voids, as a function of the separation $r$ between the cascade midpoint and the defect centre. ( $N_{\text {Vac }}$ : number of vacancies in the initial defect) 


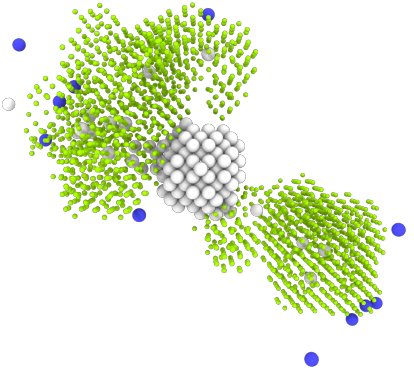

(a) $r=17 \AA$

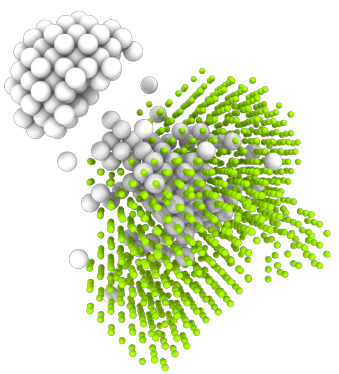

(b) $r=23 \AA$
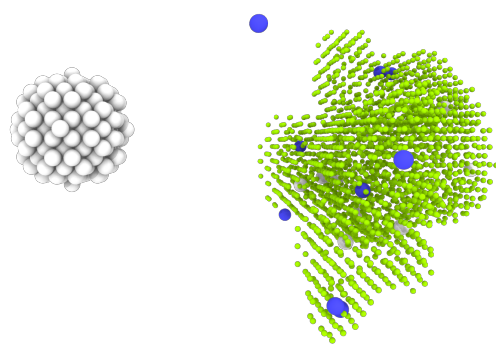

(c) $r=43 \AA$

Figure 5: Three outcomes of a cascade overlapping with a void of $N_{\mathrm{Vac}}=259$, with different separation distances $r$ between cascade center and the center of the defect. Green dots represent atoms that are classified as liquid during the maximum extent of the cascade heat spike. White spheres represent vacancies, and blue spheres represent interstitials, at the end of the simulation. 


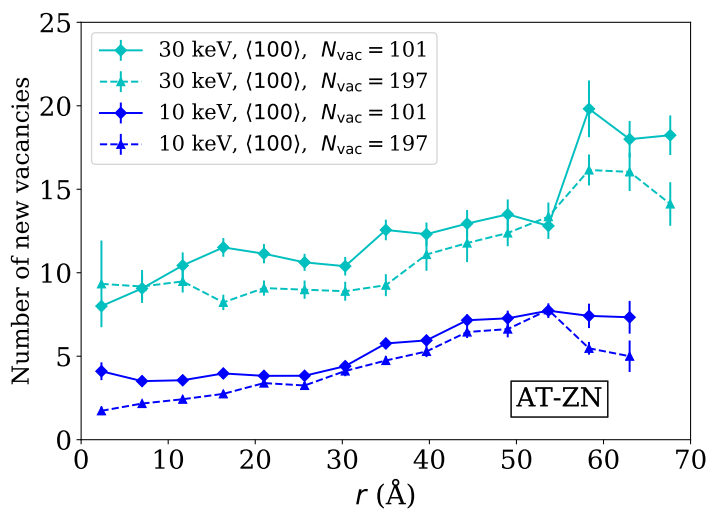

(a) $\langle 100\rangle, N_{\text {vac }}=101,197$

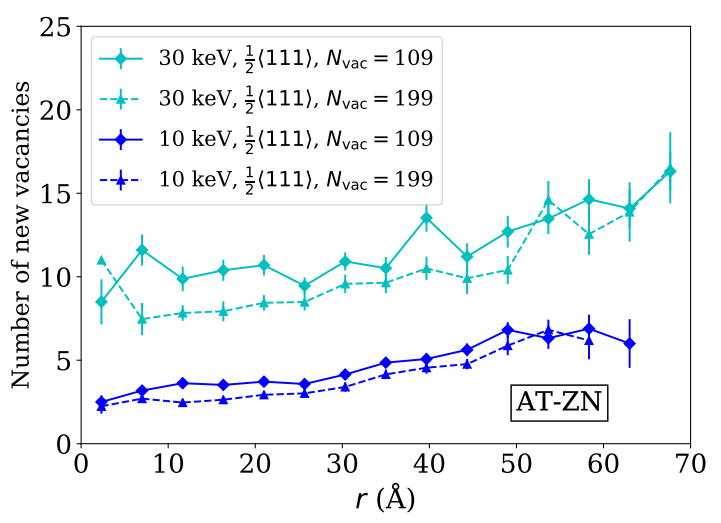

(b) $1 / 2\langle 111\rangle, N_{\text {vac }}=109,199$

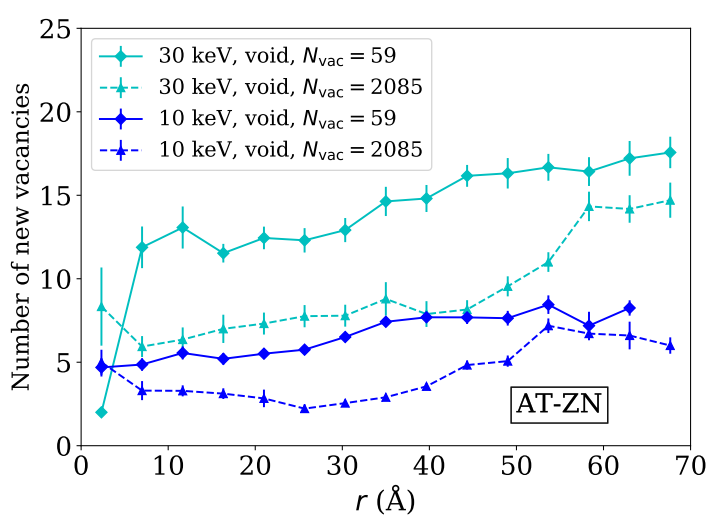

(c) void, $N_{\text {vac }}=59,2085$

Figure 6: Number of new defects created in $10 \mathrm{keV}$ and $30 \mathrm{keV}$ cascade simulations with the AT-ZN potential at $300 \mathrm{~K}$, as a function of the separation $r$ between cascade center and defect center. ( $N_{\text {vac }}$ : number df2vacancies in the initial system) 
change. For both loops and voids, the cluster size decreases the most when the cascade only partially overlaps with the defect. When the cascade fully overlaps with the defect, the average decrease in cluster size is smaller. When the cascade does not overlap with the pre-existing defect, no change in the cluster size is observed. Hence only direct or partial overlap of the cascade region with the defect affects the sizes of the pre-existing defect clusters. The difference between full and partial overlap is more pronounced for voids than for loops. This is an effect of the cascade splitting described earlier, which reduces the effect of the overlapping cascade when it is centered on a void. For both loops and voids, the maximum fractional decrease in cluster size is larger for smaller defects, as can be expected.

The effect of overlap is overall stronger for pre-existing dislocation loops than for voids, with a decrease in size of up to $60 \%$ for pre-existing vacancytype dislocation loops. This is likely due to the fact that a loop exists in nearly perfectly packed crystal, allowing a cascade at full intensity to overlap the defect, with the resulting atom deficiency in the cascade region causing a decrease in the production of new defects. On the other hand, a void, being a completely empty region, cannot support a cascade, and the energy of the recoils hence spreads further around the defect, rather than enveloping it in a dense cascade.

For loops, our results show a strong dependence on the interatomic potential, with the strongest effect of decreasing loop size seen with the DND-BN potential, and only a very slight effect with the AT-ZN potential. This is related to the ability of the potential to form dislocations upon recrystallization of the cascade core, as will be discussed in the following sections.

\subsection{Change in defect morphology}

A cascade overlapping with an existing defect has the potential to change the morphology of the defect completely. In many cases, the resulting defect has a complicated structure [17], and determining what the resulting low-energy configuration will be after relaxation at a finite temperature is a non-trivial question, and is beyond the scope of the current work. However, the defect may also transform already during the cooling and recrystallization phase of the cascade into a well formed dislocation loop with a defined Burgers vector. In Figure 9, we plot the fraction of overlapping cascade events that result in a well-formed dislocation loop. The most striking result is the large discrepancy between the different interatomic potentials. The sensitivity of the resulting morphology to the energy landscape of the intermediate, highly distorted atomic configurations sets huge requirements on the potential.

The AT-ZN potential shows the strongest tendency to form dislocation loops rather than voids, despite correctly predicting voids to be the more energetically favourable vacancy configuration (see Appendix and Ref. [37]). For the predictions of this potential, we identify two distinct mechanisms of loop formation from cascade overlap, resulting in different final Burgers vectors. In full overlap of the cascade with a pre-existing loop, the end product is most likely a dislocation loop with Burgers vector $\langle 100\rangle$, irrespective of the Burgers vector of the initial loop. For full overlap with voids, the frequency of formation of a 


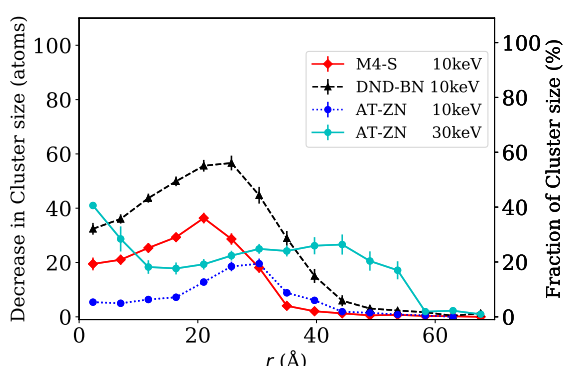

(a) $\langle 100\rangle, N_{\text {vac }}=101$

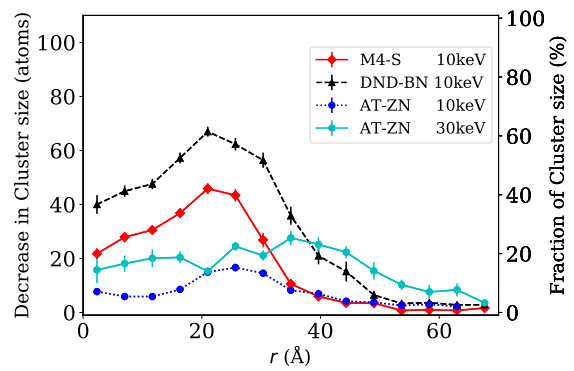

(c) $1 / 2\langle 111\rangle, N_{\text {vac }}=109$

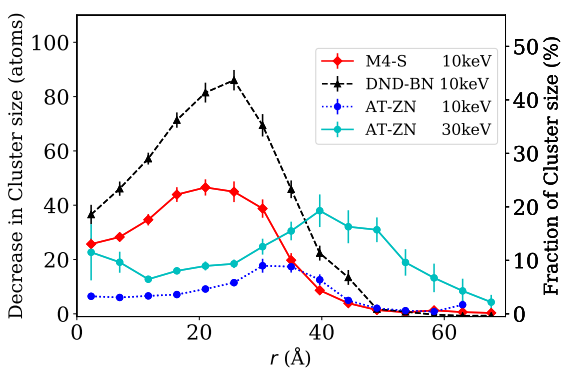

(b) $\langle 100\rangle, N_{\text {vac }}=197$

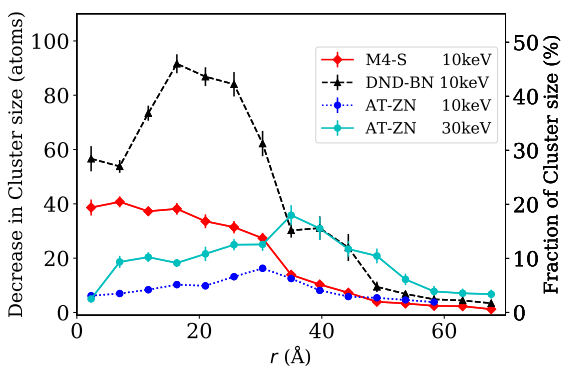

(d) $1 / 2\langle 111\rangle, N_{\text {vac }}=199$

Figure 7: The average change in size of the pre-existing dislocation loop (in terms of the largest surviving cluster) after an overlapping cascade, as a function of the distance between defect and cascade centers. 


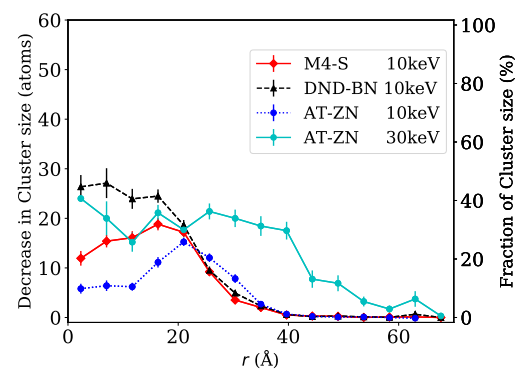

(a) void, $N_{\text {vac }}=59$

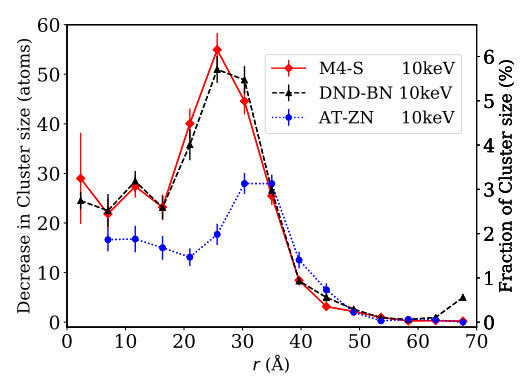

(c) void, $N_{\text {vac }}=893$

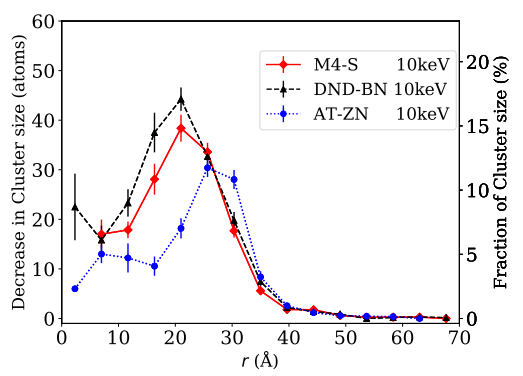

(b) void, $N_{\text {vac }}=259$

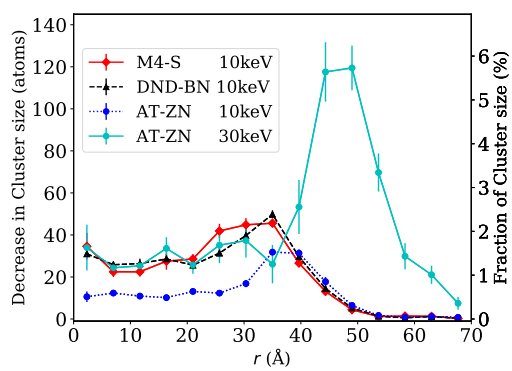

(d) void, $N_{\text {vac }}=2085$

Figure 8: The average change in size of the pre-existing void (in terms of the largest surviving cluster) after an overlapping cascade, as a function of the distance between defect and cascade centers.

dislocation loop is lower, but if a loop is formed, it has Burgers vector $\langle 100\rangle$. In these overlap events, the initial defect is fully enveloped, and all initial structure is destroyed by the cascade. As the cascade cools, the lattice recrystallizes independently of the pre-existing defect structure. With the AT-ZN potential, the recrystallization dynamics are such that they favour the formation of a $\langle 100\rangle$ loop, despite this structure having a higher formation energy than the $1 / 2\langle 111\rangle$ loop (see Appendix and Ref. [37]). This effect is observed less often for larger loops than for smaller ones, since the probability of the cascade fully destroying the initial structure decreases as the loop size increases.

As the overlap distance increases, and cascades overlap only partially with the pre-existing defect, the prevalence of $\langle 100\rangle$ loops decreases. For overlap with a $1 / 2\langle 111\rangle$ loop, the final product quickly becomes dominated by $1 / 2\langle 111\rangle$ loops. For overlap with $\langle 100\rangle$ loops, on the other hand, $\langle 100\rangle$ loops are still a possible outcome of partial overlap events, but the frequency of $1 / 2\langle 111\rangle$ loops increases at the point where the cascade destroys only part of the preexisting dislocation loop. In these cases, the dislocation line closes again upon 


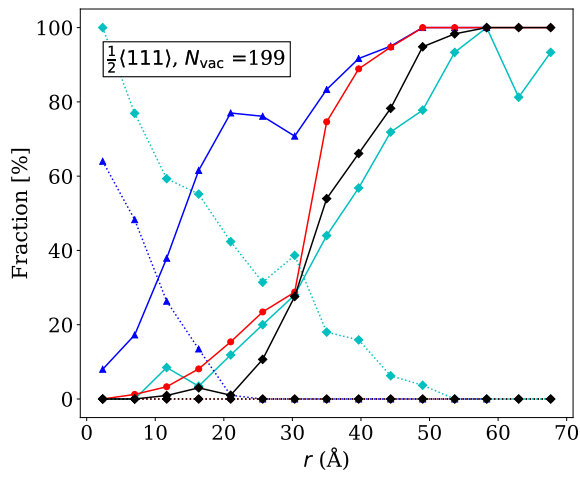

-.* (100) DND-BN, $10 \mathrm{keV}$ $\multimap \frac{1}{2}(111)$ DND-BN, $10 \mathrm{keV}$ ..... (100) M4-S, $10 \mathrm{keV}$ $-\frac{1}{2}(111) \mathrm{M} 4-\mathrm{S}, \quad 10 \mathrm{keV}$ 4... (100) AT-ZN, $10 \mathrm{keV}$ $+\frac{1}{2}(111)$ AT-ZN, $10 \mathrm{keV}$ -.. (100) AT-ZN, $30 \mathrm{keV}$ $\downarrow-\frac{1}{2}(111)$ AT-ZN, $30 \mathrm{keV}$

(a) $1 / 2\langle 111\rangle, N_{\mathrm{vac}}=199$

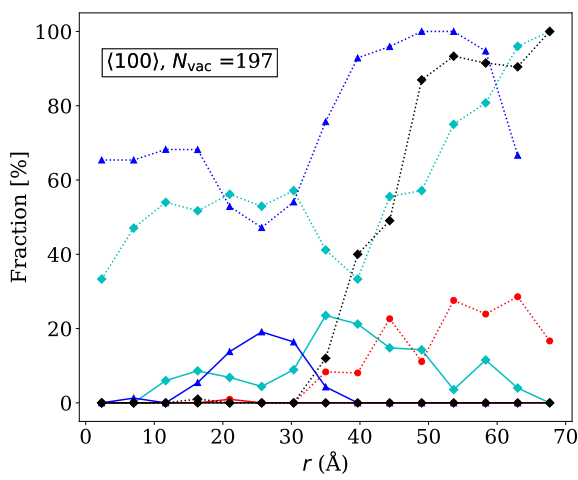

†. (100) DND-BN, $10 \mathrm{keV}$ $\multimap \frac{1}{2}(111)$ DND-BN, $10 \mathrm{keV}$ .... (100) M4-S, $\quad 10 \mathrm{keV}$ $\rightarrow \frac{1}{2}(111) \mathrm{M} 4-\mathrm{S}, \quad 10 \mathrm{keV}$ $\ldots$... $(100)$ AT-ZN, $10 \mathrm{keV}$ $+\frac{1}{2}(111)$ AT-ZN, $10 \mathrm{keV}$ +... (100) AT-ZN, $30 \mathrm{keV}$ $\leftarrow \frac{1}{2}(111)$ AT-ZN, $30 \mathrm{keV}$

(b) $\langle 100\rangle, N_{\text {vac }}=197$

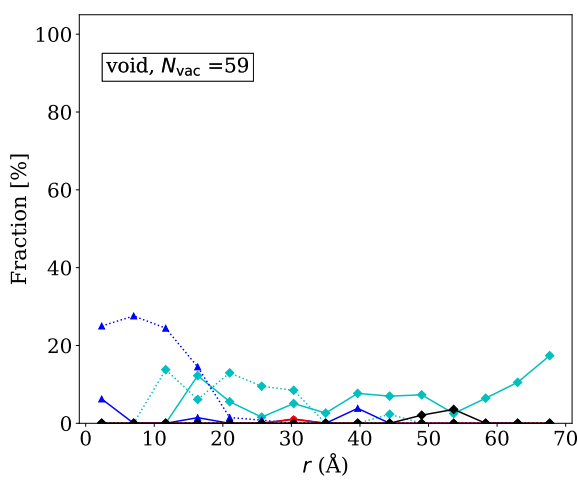

-... (100) DND-BN, $10 \mathrm{keV}$ $\leftarrow \frac{1}{2}(111)$ DND-BN, $10 \mathrm{keV}$ क. (100) M4-S, $10 \mathrm{keV}$ $\rightarrow \frac{1}{2}(111) \mathrm{M} 4 \mathrm{-S}, \quad 10 \mathrm{keV}$ … (100) AT-ZN, $10 \mathrm{keV}$ - $\frac{1}{2}(111)$ AT-ZN, $10 \mathrm{keV}$ -100) AT-ZN, $30 \mathrm{keV}$ $\nmid \frac{1}{2}\{111)$ AT-ZN, $30 \mathrm{keV}$

(c) void, $N_{\mathrm{vac}}=59$

Figure 9: Fraction of cascades producing dislocation loops in the surviving damage, for three different initial defects. 
recrystallization, but frequently the new segment has Burgers vector $1 / 2\langle 111\rangle$. This mixed dislocation subsequently relaxes to a full $1 / 2\langle 111\rangle$ loop. For overlap with voids, only very few cases of dislocation formation are observed for partial overlap, but in all cases the loop that forms has Burgers vector $1 / 2\langle 111\rangle$. As the distance between the pre-existing defect and the cascade increases further, the defect is left intact, except for pre-existing $\langle 100\rangle$ loops with the M4-S potential, for which the $\langle 100\rangle$ loop is unstable under the disruption by a closely occurring cascade. For all potentials, the cascade can form more complex loop structures during partial overlap, consisting of multiple loop segments, or vacancy clusters with dislocation segments terminating in the disordered region.

Overlap with voids show significantly less tendency to form dislocation loops than overlap with loops, since the number of vacancies contained in the voids would require a significantly expanded cascade region in order to accommodate a full dislocation loop with the same atom count. However, in the AT-ZN potential one could sometimes observe dislocation loops forming on the side of the void. Figure 10 shows an example of an almost complete dislocation loop joined to a void, formed with the AT-ZN potential.

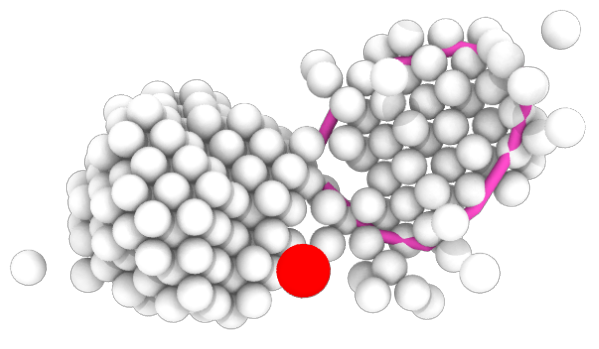

Figure 10: A $\langle 100\rangle$ loop formed on the side of a void $\left(N_{\mathrm{vac}}=259\right)$ by a $10 \mathrm{keV}$ cascade, with the AT-ZN potential. White spheres: vacancies, red spheres: interstitials, magenta line: $\langle 100\rangle$ dislocation.

In Figure 11, we show a time series of a representative case where a $1 / 2\langle 111\rangle$ loop changes to a $\langle 100\rangle$ loop with the AT-ZN potential. The initial $1 / 2\langle 111\rangle$ loop is fully destroyed by the overlapping cascade, with no identifiable dislocation segments remaining. As the disordered region crystallizes, complex dislocation structures can be identified, which eventually stabilize as a fully formed 

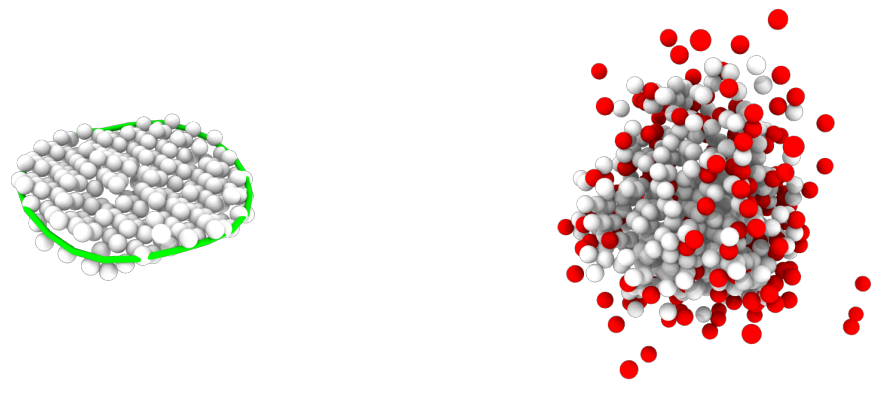

(a) time $\approx 0 \mathrm{fs}$

(b) time $\approx 550 \mathrm{fs}$
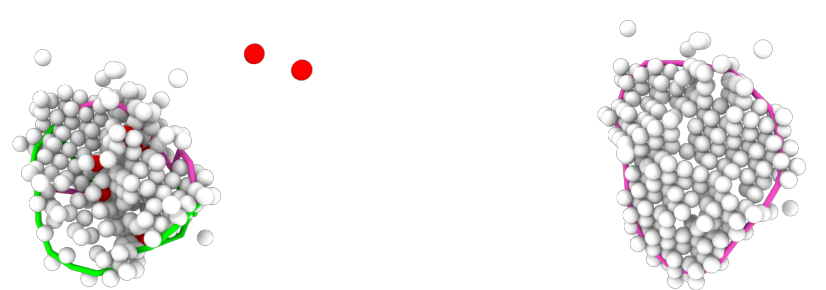

(c) time $\approx 24 \mathrm{ps}$

(d) time $\approx 50 \mathrm{ps}$

Figure 11: Destruction and reformation of a dislocation loop with the AT-ZN potential, by a $10 \mathrm{keV}$ cascade on a $1 / 2\langle 111\rangle, N_{\text {vac }}=199$ loop, white: vacancies, red: interstitials, green lines: $1 / 2\langle 111\rangle$ and magenta lines: $\langle 100\rangle$

$\langle 100\rangle$ loop.

\section{Discussion}

We find that dislocation loops decrease the numbers of new defects more efficiently than voids at full overlap. This is partly explained by the fact that voids are observed to induce cascade splitting, even though these PKA energies are otherwise well below the subcascade splitting threshold in $\mathrm{W}[32,38]$. Hence the final defects are effectively the products of two separate cascade regions. In this PKA energy range, the defect production from independent cascades has been shown to follow a power law of the form $2.21\left(E_{\mathrm{MD}}\right)^{0.74}$ [39]. Hence, splitting the cascade into two separate regions with a combined energy of $10 \mathrm{keV}$ can potentially result in $20 \%$ more defects than from a single $10 \mathrm{keV}$ cascade, not considering the stochastic variations between individual cascades. Cascade splitting is observed only when the PKA is initiated close enough to the void that it has enough kinetic energy when it enters the void to give rise to a second cascade region on the other side of the void. Hence this effect occurs only in 
cases of full overlap, while for partial overlap the reduction in defect numbers is similar for similarly sized loops and voids.

The observed persistent decrease in vacancy cluster size as a result of an overlapping cascade, in contrast to the case for interstitial defects, is a natural consequence of the different formation mechanisms of interstitial and vacancy clusters in cascades. The former are formed from dense regions of atoms ejected or pushed to the perimeter of the cascade region [40], while vacancies are pushed towards the center of the cascade as the recrystallization front progresses inwards while the cascade region cools [41]. As a result of this inwards migration of vacancies, the overlapping cascade splits pre-existing vacancy clusters most efficiently when the cascade partially overlaps with the defect, leaving part of it in its original position, and pulling part of it towards the center of the secondary cascade. This is seen as a clear peak in the decrease of void size shown in Figure 7. This mechanism of void destruction presents one of the underlying reasons, in addition to vacancy mobility, for differences in void formation observed between neutron $[42,43]$ and ion $[44,31,45]$ irradiation, through efficient destruction of voids relative to the thermally driven void growth, in the higher dose rate of ion irradiation experiments.

We observe two different formation mechanisms of dislocation loops, resulting in loops with different Burgers vectors. In full overlap, the cascade core has the potential to recrystallize to a defect configuration that is not determined by the lowest formation energy. On the other hand, where parts of the initial defect survive, subsequent evolution of a 2-part defect morphology leads to lower energy configurations, clearly favouring the loop with a Burgers vector involving the lowest formation energy. This is in contrast to cascade overlap with interstitial clusters, where the lowest-energy defect configuration is the most likely product in both full and partial overlap, although higher-energy configurations were also formed with significant probabilities [18]. Hence, "energetically unfavourable" defect configurations may become more frequent in neutron irradiated tungsten after the onset of cascade overlap, but depend on the relation between the size of the defect and the diameter of the cascade, through the requirement that the initial defect be fully enveloped by the cascade. However, this prediction is strongly dependent on the interatomic potential, and it is not clear which potential provides a physically sound prediction, due to the challenges associated with benchmarking a potential for the highly non-equilibrium conditions and distorted atomic configurations that occur during cascade core recrystallization. Nevertheless, the AT-ZN potential represents the most 'generic' potential of the ones that were employed in this study. The DND-BN and M4-S potentials have an increasing number of fitted parameters, introduced through the cubic knot functions used to represent the various energy contributions in the potentials. This serves to improve predictions for certain targeted properties, but simultaneously presents higher challenges for transferability. Cascade dynamics involves an extremely wide range of states of the material, from the initial ground state lattice, to an under-dense core and over-dense outer layer in the heat spike formation phase, to the subsequent liquid-like core which persists at temperatures in excess of $10000 \mathrm{~K}$, and finally the recrystallization process 
involving the interface between the lattice and the liquid core, and the defect structures that emerge from this state. As such, it can be expected that the more generic potential provides the most comprehensively accurate prediction. Furthermore, the prediction of $\langle 100\rangle$-type dislocation loops in irradiated $\mathrm{W}$ is in agreement with experimental observations [31], increasing the level of confidence in the AT-ZN potential, which alone out of the three potentials tested here has predicted the in-cascade formation of $\langle 100\rangle$-type loops of both vacancy and interstitial nature [46].

The tendency of overlapping cascades to reduce vacancy cluster sizes will affect predictions of damage evolution, compared to calculations based solely on vacancy mobility, and stability and growth rates of defects. This implies that cascade overlap must be taken into account when modelling microstructural evolution under irradiation, whether the employed methods are based on mean field calculations or OKMC. Furthermore, this mechanism will serve to inhibit void formation in the limits of high dose rate or low temperature, which are typical conditions in ion irradiation experiments. This effect, together with the void-induced cascade splitting, has profound implications for the comparison of neutron and ion irradiated materials. Since higher energy cascades typically form larger clusters [47, 32], void-induced cascade splitting will also shift the defect production towards smaller, more mobile defects, hence enhancing the possibility of recombination as compared to prolonged irradiation under conditions that do not favour void formation. This implies that a simple shift in temperature necessarily cannot compensate for dose rate effects in neutron versus ion irradiation experiments, if the underlying microstructure is different. 


\section{Conclusions}

We have shown that the defect production from collision cascades in tungsten is decreased by the presence of pre-existing vacancy-type defects, with a functional dependence on the degree of overlap very similar to that of overlap with interstitial defects.

However, we find qualitatively different behaviour in many respects, compared to overlap with interstitial defects. We find that overlapping cascades consistently decrease the size of the pre-existing defects, with the strongest effect seen when cascades partially overlap with the defect. Further, we observe void-induced cascade splitting for cascade energies well below the subcascade splitting threshold in tungsten. These effects will cause clear differences not described by standard rate theory models in damage accumulation under irradiation conditions that favour void formation, i.e. where vacancies are mobile and dose rates low, as compared to the limit of high dose rate and low temperature.

In addition, we identify two distinct mechanisms of loop formation resulting from an overlapping cascade, neither one showing dependence on the original defect structure. On the one hand, when cascades fully envelop a defect, the final defect is not constrained by considerations of formation energy. Rather, we observe frequent formation of $\langle 100\rangle$ type dislocation loops in such scenarios, even though the interatomic potential predicts a higher formation energy for $\langle 100\rangle$ than $1 / 2\langle 111\rangle$ loops. In contrast, when cascades only partially overlap a dislocation loop, the resulting - frequently 2-part - structure often relaxes to a lower energy loop during the cascade relaxation time, i.e. on the order of picoseconds.

While the different potentials considered in this work show agreement regarding the efficiency of production of new defects from overlapping cascades, they differ significantly with regards to predictions of the structure of the final defects. In particular, potentials disagree on the readiness to form dislocation structures during recrystallization of the heat spike. The different loop forma-

tion mechanisms identified in this work are found based on the predictions of the AT-ZN potential, which shows the strongest tendency to form dislocations. We conclude that more accurate potentials are needed for quantitatively reliable predictions of radiation defect morphology.

\section{Acknowledgements}

This work has been carried out within the framework of the EURO- fusion Consortium and has received funding from the Euratom research and training programme 2014-2018 and 2019-2020 under grant agreement No. 633053. The views and opinions expressed herein do not necessarily reflect those of the European Commission. AES acknowledges support from the Academy of Finland through project No. 311472. We acknowledge grants of computer capacity from the IT Centre for Science in Finland, CSC and the Finnish Grid and Cloud Infrastructure (persistent identifier urn:nbn:fi:research-infras-2016072533 ). 


\section{Appendix A. Formation energies of vacancy clusters}

Figure A.12 shows the formation energies of different vacancy-type clusters in $\mathrm{W}$ as predicted by the different interatomic potentials. The formation energies were calculated following a conjugate gradient minimization of systems containing a dislocation loop or spherical void of a given size at zero pressure. The lines connecting the data points are fits to the corresponding scaling law of dislocation loops or voids, discussed in [36]. The formation energy of dislocation loops scales as

$$
E_{\mathrm{f}}=a_{0} \sqrt{N} \ln (N)+a_{1} \sqrt{N}+a_{2},
$$

where $N$ is the number of vacancies in the loop, and $a_{i}$ are used as fitting parameters. For voids, the formation energy scales as [36]

$$
E_{\mathrm{f}}=a N^{2 / 3} .
$$

All potentials predict the same order of stability for the different clusters at large sizes, with voids lowest and $\langle 100\rangle$ loops highest in energy. The differences in energy between the two loop types are small in the DND-BN and M4-S potentials, and at small sizes $\langle 100\rangle$ loops are lower in energy.

\section{References}

[1] M. Rieth, S. Dudarev, S. G. de Vicente, J. Aktaa, T. Ahlgren, S. Antusch, D. Armstrong, M. Balden, N. Baluc, M.-F. Barthe, W. Basuki, M. Battabyal, C. Becquart, D. Blagoeva, H. Boldyryeva, J. Brinkmann, M. Celino, L. Ciupinski, J. Correia, A. D. Backer, C. Domain, E. Gaganidze, C. García-Rosales, J. Gibson, M. Gilbert, S. Giusepponi, B. Gludovatz, H. Greuner, K. Heinola, T. Höschen, A. Hoffmann, N. Holstein, F. Koch, W. Krauss, H. Li, S. Lindig, J. Linke, C. Linsmeier, P. López-Ruiz, H. Maier, J. Matejicek, T. Mishra, M. Muhammed, A. Muñoz, M. Muzyk, K. Nordlund, D. Nguyen-Manh, J. Opschoor, N. Ordás, T. Palacios, G. Pintsuk, R. Pippan, J. Reiser, J. Riesch, S. Roberts, L. Romaner, M. Rosiński, M. Sanchez, W. Schulmeyer, H. Traxler, A. Ureña, J. van der Laan, L. Veleva, S. Wahlberg, M. Walter, T. Weber, T. Weitkamp, S. Wurster, M. Yar, J. You, A. Zivelonghi, Journal of Nuclear Materials 432 (2013) $482-500$.

[2] V. Philipps, Journal of Nuclear Materials 415 (2011) S2 - S9. Proceedings of the 19th International Conference on Plasma-Surface Interactions in Controlled Fusion.

[3] H. Bolt, V. Barabash, G. Federici, J. Linke, A. Loarte, J. Roth, K. Sato, Journal of Nuclear Materials 307-311 (2002) $43-52$.

[4] K. Nordlund, S. J. Zinkle, A. E. Sand, F. Granberg, R. S. Averback, R. Stoller, T. Suzudo, L. Malerba, F. Banhart, W. J. Weber, F. Willaime, S. Dudarev, D. Simeone, J. Nucl. Mater. 512 (2018) 450-479. 

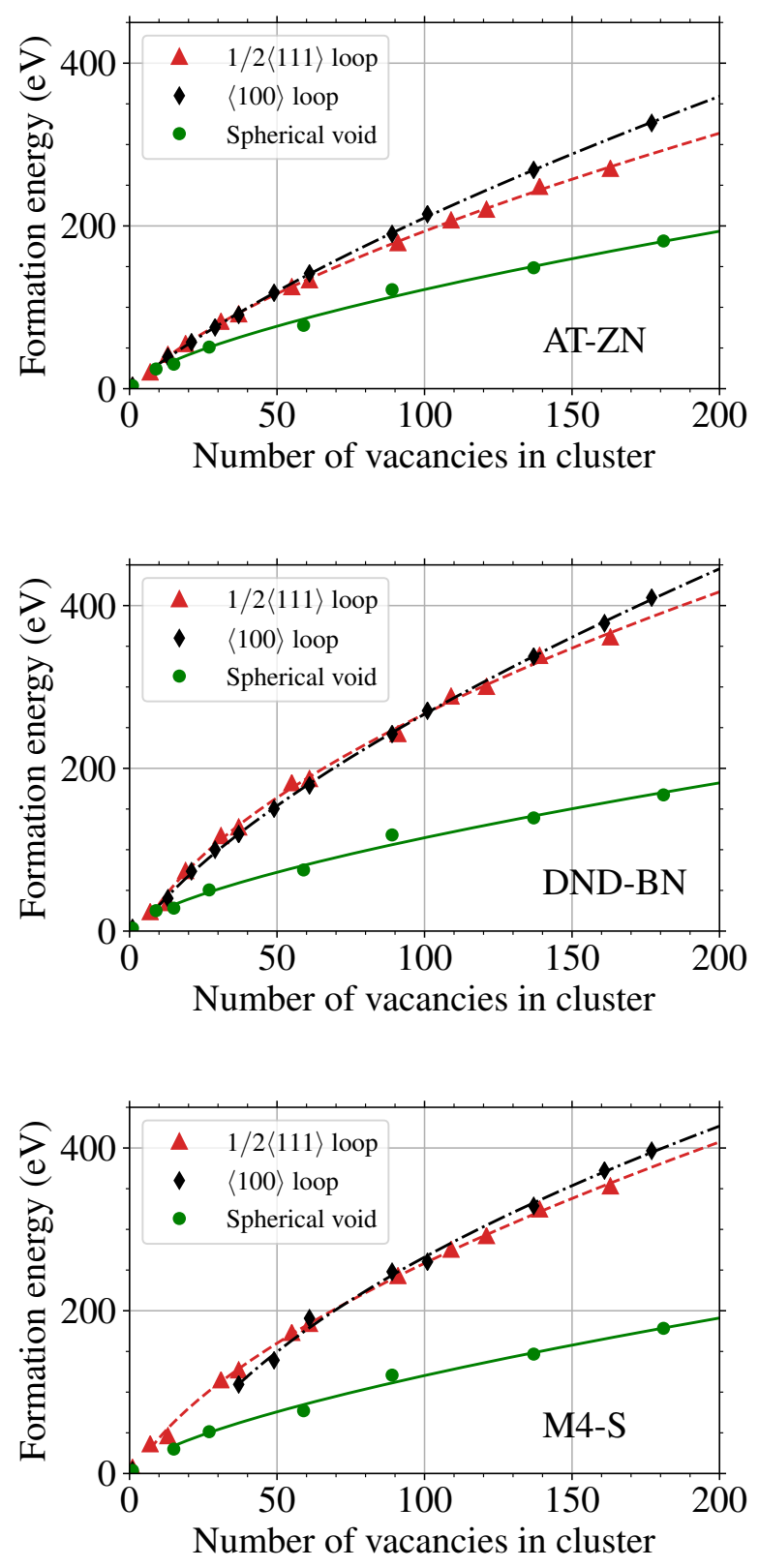

Figure A.12: Formation energies of vacancy clusters predicted by the different interatomic potentials 
[5] K. Nordlund, S. J. Zinkle, T. Suzudo, R. S. Averback, A. Meinander, F. Granberg, L. Malerba, R. Stoller, F. Banhart, B. Weber, F. Willaime, S. Dudarev, D. Simeone, Primary radiation damage in materials: Review of current understanding and proposed new standard displacement damage model to incorporate in-cascade mixing and defect production efficiency effects, OECD Nuclear Energy Agency, Paris, France, 2015.

[6] A. J. E. Foreman, W. J. Phythian, C. A. English, Rad. Eff. \& Def. in Sol. 129 (1994) 25.

[7] F. Gao, D. J. Bacon, A. F. Calder, P. E. J. Flewitt, T. A. Lewis, J. Nucl. Mater. 230 (1996) 47.

[8] K. Nordlund, R. S. Averback, Phys. Rev. B 56 (1997) 2421-2431.

[9] D. Terentyev, K. Vörtler, C. Björkas, K. Nordlund, L. Malerba, J. Nucl. Mater. 417 (2011) 1063-1066.

[10] J. Nord, K. Nordlund, J. Keinonen, Nucl. Instr. Meth. Phys. Res. B 193 (2002) 294-298.

[11] F. Granberg, K. Nordlund, M. W. Ullah, K. Jin, C. Lu, H. Bei, L. M. Wang, F. Djurabekova, W. J. Weber, , Y. Zhang, Phys. Rev. Lett. 116 (2016) 135504.

[12] E. Levo, F. Granberg, C. Fridlund, K. Nordlund, F. Djurabekova, J. Nucl. Mater. 490 (2017) 323.

[13] A. Dunlop, B. Pande, K. Böning, P. Rosner, H. Schaefer, Journal of Nuclear Materials 108-109 (1982) 83-94.

[14] I. Robertson, M. Kirk, W. E. King, Scripta Metallurgica 18 (1984) 317 320.

[15] F. Gao, D. Bacon, A. Calder, P. Flewitt, T. Lewis, Journal of Nuclear Materials 230 (1996) $47-56$.

[16] F. Granberg, J. Byggmästar, A. E. Sand, K. Nordlund, EPL (Europhysics Letters) 119 (2017) 56003.

[17] A. Sand, J. Byggmästar, A. Zitting, K. Nordlund, Journal of Nuclear Materials 511 (2018) $64-74$.

[18] J. Byggmästar, F. Granberg, A. E. Sand, A. Pirttikoski, R. Alexander, M.-C. Marinica, K. Nordlund, Journal of Physics: Condensed Matter 31 (2019) 245402.

[19] G. Nandipati, W. Setyawan, H. L. Heinisch, K. J. Roche, R. J. Kurtz, B. D. Wirth, J. Nucl. Mater. 462 (2015) $338-344$. 
[20] N. Castin, A. Bakaev, G. Bonny, A. Sand, L. Malerba, D. Terentyev, Journal of Nuclear Materials 493 (2017) 280-293.

[21] C. Becquart, C. Domain, U. Sarkar, A. DeBacker, M. Hou, J. Nucl. Mater. 403 (2010) 75-88.

[22] H. J. Berendsen, J. v. Postma, W. F. van Gunsteren, A. DiNola, J. Haak, The Journal of chemical physics 81 (1984) 3684-3690.

[23] K. Nordlund, 2010. PARCAS computer code. The main principles of the molecular dynamics algorithms are presented in [35? ]. The adaptive time step and electronic stopping algorithms are the same as in [? ].

[24] M. S. Daw, M. I. Baskes, Phys. Rev. B 29 (1984) 6443-6453.

[25] P. M. Derlet, D. Nguyen-Manh, S. L. Dudarev, Phys. Rev. B 76 (2007) 054107.

[26] C. Björkas, K. Nordlund, S. Dudarev, Nuclear Instruments and Methods in Physics Research Section B: Beam Interactions with Materials and Atoms 267 (2009) 3204 - 3208. Proceedings of the Ninth International Conference on Computer Simulation of Radiation Effects in Solids.

[27] G. J. Ackland, R. Thetford, Philosophical Magazine A 56 (1987) 15-30.

[28] Y. Zhong, K. Nordlund, M. Ghaly, R. S. Averback, Phys. Rev. B 58 (1998) 2361-2364.

[29] M.-C. Marinica, L. Ventelon, M. R. Gilbert, L. Proville, S. L. Dudarev, J. Marian, G. Bencteux, F. Willaime, Journal of Physics: Condensed Matter 25 (2013) 395502.

[30] A. E. Sand, J. Dequeker, C. S. Becquart, C. Domain, K. Nordlund, Journal of Nuclear Materials 470 (2016) 119-127.

[31] X. Yi, M. L. Jenkins, K. Hattar, P. D. Edmondson, S. G. Roberts, Acta Materialia 92 (2015) $163-177$.

[32] A. E. Sand, D. R. Mason, A. D. Backer, X. Yi, S. L. Dudarev, K. Nordlund, Materials Research Letters 5 (2017) 357-363.

[33] A. Stukowski, Modelling and Simulation in Materials Science and Engineering 18 (2010) 015012.

[34] A. Stukowski, K. Albe, Modelling and Simulation in Materials Science and Engineering 18 (2010) 085001.

[35] K. Nordlund, M. Ghaly, R. S. Averback, M. Caturla, T. Diaz de la Rubia, J. Tarus, Phys. Rev. B 57 (1998) 7556-7570.

[36] M. R. Gilbert, S. L. Dudarev, P. M. Derlet, D. G. Pettifor, J. Phys.: Condens. Matter 20 (2008) 345214. 
[37] J. Fikar, R. Schäublin, D. R. Mason, D. Nguyen-Manh, Nuclear Materials and Energy 16 (2018) $60-65$.

[38] A. D. Backer, A. E. Sand, K. Nordlund, L. Luneville, D. Simeone, S. L. Dudarev, EPL (Europhysics Letters) 115 (2016) 26001.

[39] W. Setyawan, A. P. Selby, N. Juslin, R. E. Stoller, B. D. Wirth, R. J. Kurtz, Journal of Physics: Condensed Matter 27 (2015) 225402.

[40] J. A. Brinkman, J. Appl. Phys. 25 (1954) 961.

[41] K. Nordlund, R. S. Averback, Phys. Rev. B 59 (1999) 20-23.

[42] A. Hasegawa, M. Fukuda, S. Nogami, K. Yabuuchi, Fusion Engineering and Design 89 (2014) 1568 - 1572.

[43] X. Hu, T. Koyanagi, M. Fukuda, N. K. Kumar, L. L. Snead, B. D. Wirth, Y. Katoh, Journal of Nuclear Materials 480 (2016) $235-243$.

[44] X. Yi, M. L. Jenkins, M. B. no, S. G. Roberts, Z. Zhou, M. A. Kirk, Phil. Mag. A 93 (2013) 1715-1738.

[45] X. Yi, M. L. Jenkins, M. A. Kirk, Z. Zhou, S. G. Roberts, Acta Materialia 112 (2016) $105-120$.

[46] A. E. Sand, S. L. Dudarev, K. Nordlund, EPL 103 (2013) 46003.

[47] W. Setyawan, G. Nandipati, K. J. Roche, H. L. Heinisch, B. D. Wirth, R. J. Kurtz, Journal of Nuclear Materials 462 (2015) 329 - 337. 